\title{
Dynamics Analysis for rigid-flexible coupling systems of a Planar Flexible Beam in Non-inertial Reference Frame
}

\author{
Yehao Yan ${ }^{a}$, Xingsuo $\mathrm{He}^{\mathrm{b}}$ and Fengyan Deng ${ }^{\mathrm{c}}$ \\ Department of Engineering Mechanics, Northwestern Polytechnical University, Xi'an, 710072, \\ China. \\ ayyhyyahao275@163.com, bxingsuoh@nwpu.edu.cn, c2739078107@qq.com
}

\begin{abstract}
Keywords: large overall motion, nonlinear deformation, planar flexible beam, non-inertial reference frame, the precise coupling model.
\end{abstract}

\begin{abstract}
In this paper, the dynamic properties of a planar flexible beam with large overall motion and nonlinear deformation in non-inertial reference frame are investigated. The dynamic response of the present model is compared with that of zero-order approximate model and one-order coupling model. Then changing of dynamic stiffening terms due to the new coupling terms is discussed according to different models. At the same time, the effect of initial static deformation in the tip is considered to study the vibrant deformation of flexible beam. In addition, when the overall motions are free, the rigid-flexible coupling dynamics theory is extend to spatial structure form planar structure. The difference among zero-order approximate model, one-order coupling model and the precise coupling model is revealed by the frequency spectrum analysis method and concludes that the speed of overall motion is a vital cause for the difference among different models. And the dynamic stiffening phenomenon still exists in rigid-flexible coupling system while the overall motion is free. But the effect of dynamic stiffening in the present exact model is not as severe as that of the oneorder coupling model.
\end{abstract}

\section{Introduction}

Dynamic modeling of flexible beams with large overall motion and nonlinear deformation is studied $[1,2,3]$. But in the flexible multibody system dynamics study, in view of the motion law of known of the flexible manipulator, a high-speed rotation of the helicopter rotor blades and the law of a given rotational ontology-satellite antenna structure, analysis of the dynamic properties of flexible body under non-inertial system is often called. Since the Kane revealed for the first time this kind of problem with the dynamic characteristics of players, some scholars use different modeling methods, consider different models, and study the flexible structures of the given motion patterns under different non-inertial systems, and on the basis of this, the multi-body system such as flexible manipulator is studied $[4,5]$.

But most of previous studies [4] by using the zero-order approximation model, taking into account the additional stiffness or deformation constraint coupling model, or one-order coupling model, focused on the research dynamic players effects as well as the flexible structure in motion under the condition of system stability and convergence. In the case of flexible deformation, the corresponding simplified processing is carried out. But as literature [6] pointed out that using the deformation model simplified processing model, the movement of the non-inertial coordinate system simulation of flexible structure, can cause the certain error. It is therefore necessary to study such problems using the precise coupling model proposed in this paper.

\section{The dynamic equation of the flexible beam under the non-inertial system}

According to the reference literature [1], the structural dynamics equations of flexible beams in non-inertial coordinates are first exported. In these kinds of problems, the large scale of motion is known, and only the node displacement is unknown. That is the coordinate of the system: $\boldsymbol{q}=\boldsymbol{q}_{f}, \dot{\boldsymbol{r}}_{0}$, $\omega$. The laws of change are known. 
When the external forces are distributed, the dynamic equations of space flexible beam are obtained.

$$
\boldsymbol{M} \ddot{\boldsymbol{q}}_{f}+\boldsymbol{G} \dot{\boldsymbol{q}}_{f}+\boldsymbol{K} \boldsymbol{q}_{f}=\boldsymbol{Q}
$$

It is a mass matrix $\boldsymbol{M}$, a gyro matrix $\boldsymbol{G}$, a stiffness matrix $\boldsymbol{K}$, and a generalized force matrix $\boldsymbol{Q}$.

Among them

$$
\begin{array}{r}
\boldsymbol{M}=\boldsymbol{M}_{33}=\boldsymbol{W}_{11}+\boldsymbol{W}_{22}+\boldsymbol{W}_{33}+\overline{\boldsymbol{W}}_{22}+\overline{\boldsymbol{W}}_{33}+\boldsymbol{W}_{44}+\overline{\overline{\boldsymbol{W}}}_{34}+\overline{\overline{\boldsymbol{W}}}_{24} \\
\boldsymbol{G}=2 \omega_{1}\left(\boldsymbol{W}_{32}-\boldsymbol{W}_{23}\right)+2 \omega_{2}\left(\boldsymbol{W}_{13}-\boldsymbol{W}_{31}\right)+2 \omega_{3}\left(\boldsymbol{W}_{21}-\boldsymbol{W}_{12}\right) \\
+\left(-\hat{\boldsymbol{W}}_{2,42}^{T}+\hat{\boldsymbol{W}}_{2,24}^{T}+\hat{\boldsymbol{W}}_{2,42}-\hat{\boldsymbol{W}}_{2,24}\right) \omega_{2}+\left(-\hat{\boldsymbol{W}}_{3,43}^{T}+\hat{\boldsymbol{W}}_{3,34}^{T}+\hat{\boldsymbol{W}}_{3,43}-\hat{\boldsymbol{W}}_{3,34}\right) \omega_{3}
\end{array}
$$

To facilitate the comparison of one-order coupling model, the stiffness matrix is written as follows.

$$
\begin{aligned}
& \boldsymbol{K}=\boldsymbol{K}_{f}+\boldsymbol{K}_{d}, \boldsymbol{K}_{d}=\boldsymbol{K}_{0}+\boldsymbol{K}_{1}
\end{aligned}
$$

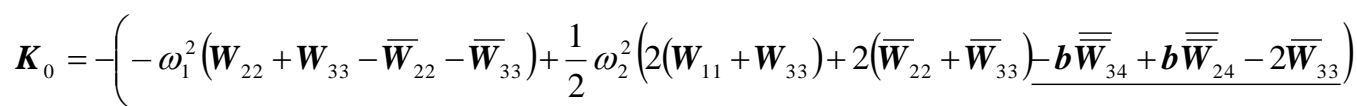

$$
\begin{aligned}
& +\frac{1}{2} \omega_{3}^{2}\left(2\left(\boldsymbol{W}_{11}+\boldsymbol{W}_{22}\right)+2\left(\overline{\boldsymbol{W}}_{22}+\overline{\boldsymbol{W}}_{33}\right)-\boldsymbol{b} \overline{\overline{\boldsymbol{W}}}_{24}+\boldsymbol{b} \overline{\overline{\boldsymbol{W}}}_{34}-2 \overline{\boldsymbol{W}}_{22}\right) \\
& +\omega_{1} \omega_{2}\left(-\boldsymbol{b} \boldsymbol{W}_{21}-\underline{\boldsymbol{b}} \overline{\boldsymbol{W}}_{02,12}+\boldsymbol{b} \hat{\boldsymbol{W}}_{2,34}+\boldsymbol{b} \hat{\boldsymbol{W}}_{3,34}\right)+\omega_{1} \omega_{3}\left(-\boldsymbol{b} \boldsymbol{W}_{31}-\boldsymbol{b} \overline{\boldsymbol{W}}_{03,13}+\boldsymbol{b} \hat{\boldsymbol{W}}_{2,24}-\boldsymbol{b} \hat{\boldsymbol{W}}_{3,24}\right) \\
& \left.+\omega_{2} \omega_{3}\left(-\boldsymbol{b} \boldsymbol{W}_{32}+\frac{1}{2}\left(\boldsymbol{b} \overline{\boldsymbol{W}}_{03,23}+\boldsymbol{b} \overline{\boldsymbol{W}}_{02,23}\right)\right)\right)+\dot{\omega}_{1}\left(\boldsymbol{W}_{32}-\boldsymbol{W}_{23}+\underline{\overline{\boldsymbol{W}}_{3,23}^{T}-\overline{\boldsymbol{W}}_{2,23}^{T}}\right) \\
& -\dot{\omega}_{2}\left(\boldsymbol{W}_{31}-\boldsymbol{W}_{13}-\overline{\boldsymbol{W}}_{3,13}^{T}-\overline{\boldsymbol{W}}_{t 3,24}^{T}+\hat{\boldsymbol{W}}_{2,42}^{T}-\hat{\boldsymbol{W}}_{2,24}^{T}\right)-\dot{\omega}_{3}\left(\boldsymbol{W}_{12}-\boldsymbol{W}_{21}-\overline{\boldsymbol{W}}_{t 2,34}^{T}+\overline{\boldsymbol{W}}_{2,12}^{T}+\hat{\boldsymbol{W}}_{3,43}^{T}-\hat{\boldsymbol{W}}_{3,34}^{T}\right) \\
& \boldsymbol{K}_{1}=\left(\omega_{2}^{2}+\omega_{3}^{2}\right) \boldsymbol{D}-\left(\ddot{r}_{01}^{\prime}-\underline{\underline{-\omega_{3}} \dot{r}_{02}^{\prime}+\omega_{2} \dot{r}_{03}^{\prime}}\right) \boldsymbol{C} \\
& \boldsymbol{Q}=\omega_{1} \omega_{2}\left(\overline{\boldsymbol{Y}}_{2}^{T}-\boldsymbol{Z}_{12}^{T}\right)+\omega_{1} \omega_{3}\left(\overline{\boldsymbol{Y}}_{3}^{T}-\boldsymbol{Z}_{13}^{T}\right)+\omega_{2}^{2} \boldsymbol{Z}_{11}^{T}+\omega_{2} \omega_{3}\left(\overline{\overline{\boldsymbol{Y}}}_{34}^{T}-\overline{\overline{\boldsymbol{Y}}}_{24}^{T}\right)+\omega_{3}^{2} \boldsymbol{Z}_{11}^{T} \\
& -\left(\boldsymbol{Y}_{1}^{T} \ddot{r}_{01}^{\prime}+\boldsymbol{Y}_{2}^{T} \ddot{r}_{02}^{\prime}+\boldsymbol{Y}_{3}^{T} \ddot{r}_{03}^{\prime}+\left(\overline{\overline{\boldsymbol{Y}}}_{34}^{T}+\overline{\overline{\boldsymbol{Y}}}_{24}^{T}\right) \dot{\omega}_{1}-\left(\overline{\boldsymbol{Y}}_{3}^{T}+\boldsymbol{Z}_{13}^{T}\right) \dot{\omega}_{2}+\left(\boldsymbol{Z}_{12}^{T}+\overline{\boldsymbol{Y}}_{2}^{T}\right) \dot{\omega}_{3}\right) \\
& +\left(\omega_{2} \boldsymbol{Y}_{3}^{T}-\omega_{3} \boldsymbol{Y}_{2}^{T}\right) \dot{r}_{01}^{\prime}+\left(\omega_{3} \boldsymbol{Y}_{1}^{T}-\omega_{1} \boldsymbol{Y}_{3}^{T}\right) \dot{r}_{02}^{\prime}+\left(\omega_{1} \boldsymbol{Y}_{2}^{T}-\omega_{2} \boldsymbol{Y}_{1}^{T}\right) \dot{r}_{03}^{\prime}
\end{aligned}
$$

In this case, the velocity and acceleration of the base point of the conjoined coordinate system are respectively $\dot{\boldsymbol{r}}_{0}^{\prime}=\boldsymbol{A}^{T} \dot{\boldsymbol{r}}_{0}, \ddot{\boldsymbol{r}}_{0}^{\prime}=\boldsymbol{A}^{T} \ddot{\boldsymbol{r}}_{0}$. Its components $\dot{\boldsymbol{r}}_{01}^{\prime}, \dot{r}_{02}^{\prime}, \ddot{r}_{03}^{\prime}, \ddot{r}_{01}^{\prime}, \ddot{r}_{02}^{\prime}, \ddot{r}_{03}^{\prime}$ are in turn. Compared with the usual model of one-order coupling model, the complete geometric nonlinear precision model takes into account the torsion of the beam and the effect of bending deformation. In the lateral deformation of the lateral direction, The distortion mode of the corresponding point in the middle line is replaced by the distortion mode of any point, which increases the line item in $\boldsymbol{M} 、 \boldsymbol{G} 、 \boldsymbol{K}_{0} 、 \boldsymbol{Q}$. In addition, the additional stiffness matrix $\boldsymbol{K}_{1}$ under the precision model contains the coupling terms of angular velocity and velocity. The double underline term indicates that the dynamic stiffness term $\boldsymbol{K}_{d}$ is not only related to the $\boldsymbol{K}_{0}$ affected by the deformation pattern; In generalized force $\boldsymbol{Q}$, it also contains the coupling of angular velocity and velocity due to the basis point motion, which is the double underline term. The zero-coupling model is not included in the additional stiffness term $\boldsymbol{K}_{1}$, and there are no underlining and double-underlined terms in each matrix.

For the structure of the plane flexible beam, the mass matrix and the gyro matrix in the dynamic equation of the non-inertial coordinate system are

$$
\boldsymbol{M}=\boldsymbol{M}_{33}=\boldsymbol{W}_{11}+\boldsymbol{W}_{22}+\overline{\boldsymbol{W}}_{22}, \boldsymbol{G}=2 \dot{\theta}\left(\boldsymbol{W}_{21}-\boldsymbol{W}_{12}\right)
$$

To facilitate the comparison of one-order coupling model, the stiffness matrix is written as follows.

$$
\begin{gathered}
\boldsymbol{K}=\boldsymbol{K}_{f}+\boldsymbol{K}_{d}, \boldsymbol{K}_{d}=\boldsymbol{K}_{0}+\boldsymbol{K}_{1} \\
\boldsymbol{K}_{0}=-\dot{\theta}^{2}\left(\boldsymbol{W}_{11}+\boldsymbol{W}_{22}+\overline{\boldsymbol{W}}_{22}\right) \underline{+\dot{\theta}^{2} \overline{\boldsymbol{W}}_{22}}+\ddot{\theta}\left(\boldsymbol{W}_{21}-\boldsymbol{W}_{12}-\overline{\boldsymbol{W}}_{21}^{T}\right) \\
\boldsymbol{K}_{1}=\dot{\theta}^{2} \boldsymbol{D}-\ddot{r}_{01}^{\prime} \boldsymbol{C}+\underline{\underline{\dot{r}_{02}^{\prime} \dot{\theta} \boldsymbol{C}}} \\
\boldsymbol{Q}=\dot{\theta}^{2} \boldsymbol{Z}_{11}^{T}-\ddot{r}_{01}^{\prime} \boldsymbol{Y}_{1}^{T}-\ddot{\boldsymbol{r}}_{02}^{\prime} \boldsymbol{Y}_{2}^{T}-\ddot{\ddot{\theta}\left(\boldsymbol{Z}_{12}^{T}+\overline{\boldsymbol{Y}}_{2}^{T}\right.} \underline{\underline{+\dot{r}_{02}^{\prime} \dot{\boldsymbol{\theta}} \boldsymbol{Y}_{1}^{T}-\dot{r}_{01}^{\prime} \dot{\boldsymbol{\theta}} \boldsymbol{Y}_{2}^{T}}}
\end{gathered}
$$




\section{The dynamic characteristics of the flexible beam under the non-inertial system}

Consider a planar flexible cantilever beam consolidation which is attached to the rigid body of a rotating center $[4,7]$. as shown in figure 1 , centrosome radius for $R$, corner for $\theta$, angular velocity as the $\dot{\theta}$, angular acceleration is $\ddot{\theta}$.

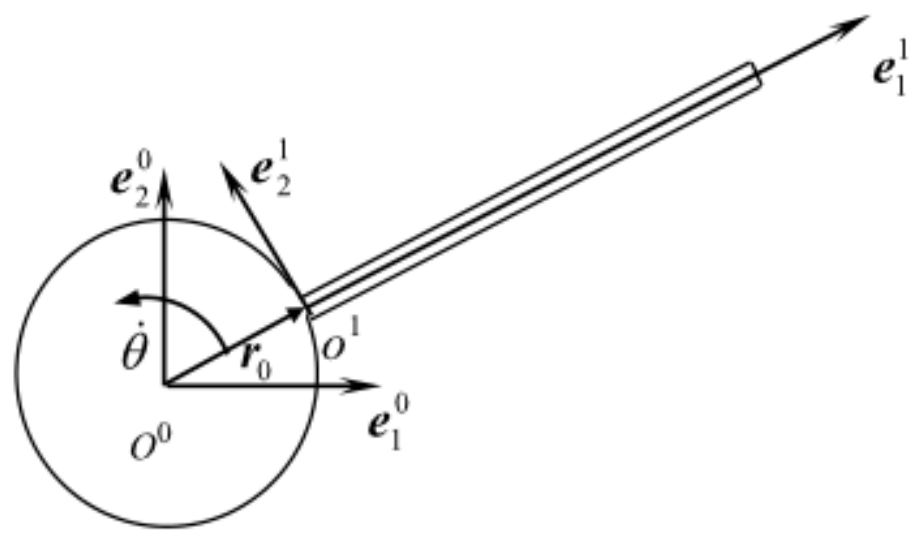

Fig. 1 A flat cantilever beam which is attached to the rigid body of a rotating center

Under the system a and the inertial system $\mathrm{e}^{0}$, the position vector of base $o^{1}$ is

$$
\begin{gathered}
\boldsymbol{r}_{0}^{\prime}=\left[\begin{array}{ll}
r_{01}^{\prime} & r_{02}^{\prime}
\end{array}\right]=\left[\begin{array}{ll}
R & 0
\end{array}\right]^{T} \\
\boldsymbol{r}_{0}=\left[\begin{array}{ll}
r_{01} & r_{02}
\end{array}\right]^{T}=\boldsymbol{A} \boldsymbol{r}_{0}^{\prime}=\left[\begin{array}{cc}
\cos \theta & -\sin \theta \\
\sin \theta & \cos \theta
\end{array}\right]\left[\begin{array}{l}
R \\
0
\end{array}\right]=\left[\begin{array}{l}
R \cos \theta \\
R \sin \theta
\end{array}\right]
\end{gathered}
$$

In the body frame $\mathrm{e}^{1}$ and the inertial system $\mathrm{e}^{0}$, the velocity vector of base $o^{1}$ is

$$
\begin{gathered}
\dot{\boldsymbol{r}}_{0}^{\prime}=\left[\begin{array}{ll}
\dot{r}_{01}^{\prime} & \dot{r}_{02}^{\prime}
\end{array}\right]^{T}=\left[\begin{array}{ll}
0 & R \dot{\theta}
\end{array}\right]^{T} \\
\dot{\boldsymbol{r}}_{0}=\left[\begin{array}{ll}
\dot{r}_{01} & \dot{r}_{02}
\end{array}\right]^{T}=\boldsymbol{A} \dot{\boldsymbol{r}}_{0}^{\prime}=\left[\begin{array}{cc}
\cos \theta & -\sin \theta \\
\sin \theta & \cos \theta
\end{array}\right]\left[\begin{array}{c}
0 \\
R \dot{\theta}
\end{array}\right]=\left[\begin{array}{c}
-R \dot{\theta} \sin \theta \\
R \dot{\theta} \cos \theta
\end{array}\right]
\end{gathered}
$$

In the body frame $\mathrm{e}^{1}$ and the inertial system $\mathrm{e}^{0}$, the acceleration vector of base $o^{1}$ is

$$
\begin{gathered}
\ddot{\boldsymbol{r}}_{0}^{\prime}=\left[\begin{array}{ll}
\ddot{r}_{01}^{\prime} & \ddot{r}_{02}^{\prime}
\end{array}\right]=\left[\begin{array}{cc}
-R \dot{\theta}^{2} & R \ddot{\theta}
\end{array}\right]^{T} \\
\ddot{\boldsymbol{r}}_{0}=\left[\begin{array}{ll}
\ddot{r}_{01} & \ddot{r}_{02}
\end{array}\right]^{T}=A \ddot{\boldsymbol{r}}_{0}^{\prime}=\left[\begin{array}{cc}
\cos \theta & -\sin \theta \\
\sin \theta & \cos \theta
\end{array}\right]\left[\begin{array}{c}
-R \dot{\theta}^{2} \\
R \ddot{\theta}
\end{array}\right]=\left[\begin{array}{l}
-R \dot{\theta}^{2} \cos \theta-R \ddot{\theta} \sin \theta \\
-R \dot{\theta}^{2} \sin \theta+R \ddot{\theta} \cos \theta
\end{array}\right]
\end{gathered}
$$

On the vertical and horizontal, the zero-coupling model directly uses the deformation model of structural dynamics, did not consider the additional stiffness $\mathrm{K} 1$, and when calculating the inherent frequency, to simplify the computation, Ignored the gyro G. The additional stiffness is the reason for the dynamic stiffness effect.

In a coupling model, the coupling terms of the base velocity and angular velocity are not considered, which the double underline term is. At the same time, in one-order coupling model, the intercoupling of vertical and horizontal deformation is not considered, so there is no Line item in $\mathrm{K}_{0}$.

For the flexible beam attached to a rotating central body, by the formula(8), (12), then

$$
\boldsymbol{K}_{1}=\dot{\theta}^{2} \boldsymbol{D}-\ddot{r}_{01}^{\prime} \boldsymbol{C}+\underline{\underline{\dot{r}_{22}^{\prime}} \dot{\boldsymbol{\theta}} \boldsymbol{C}}=\dot{\theta}^{2} \boldsymbol{D}+R \dot{\theta}^{2} \boldsymbol{C} \underline{\underline{+R \dot{\theta}^{2} \boldsymbol{C}}}
$$

By (15), it is shown that the additional stiffness increases, making the beam "rigid". But under the geometrical nonlinear precision model, considering the intercoupling of vertical and horizontal deformation, the deformation pattern is increased, and the trend of "softening" is made. The change in angular velocity is:

$$
\dot{\theta}=\left\{\begin{array}{cl}
\frac{\Omega}{T} t-\frac{\Omega}{2 \pi} \sin \frac{2 \pi t}{T} & 0<t \leq T \\
\Omega & t>T
\end{array}\right.
$$


The physical parameters are: elastic modulus is $E=6.8952 \times 10^{10} \mathrm{~N} / \mathrm{m} 2$, density is $\rho=2.766 \times 10^{3}$ $\mathrm{kg} / \mathrm{m} 3$, length is $l=8 \mathrm{~m}$, inertia moment is $I_{Z Z}=8.2189 \times 10^{-9} \mathrm{~m} 4$, and cross-sectional area is $A=7.2986 \times 10^{-5} \mathrm{~m} 2$. Because the angular velocity is known, the inertia of the central rigid body $J_{0}$ is not present in the dynamic equation of the system. Acceleration time is $T=15 \mathrm{~s}$.

1 . When the radius of centrosome $R=0 \mathrm{~m}$, by the formula (8), (12), (14), the coupling term of velocity and angular velocity is no effect on additional stiffness influence, and accelerations of points is also no effect on the additional stiffness.

When the flexible beam has no initial deformation, the initial condition is $\mathrm{q}_{f}=0, \dot{\mathrm{q}}_{f}=0$. Given the different steady speed of $\Omega=2 \mathrm{rad} / \mathrm{s}, \Omega=4 \mathrm{rad} / \mathrm{s}, \Omega=8 \mathrm{rad} / \mathrm{s}$, in this paper, using zero-order approximate model, one-order coupling model and the precise coupling model, point distortion displacement are calculated separately. When the speed was raised, the simulation results of the zeroorder approximate model diverged. At different speeds, one-order coupling model and the precise model of this paper are very small, and the simulation results converge. This shows that the newly added coupling $\overline{\mathrm{W}}_{22}, \overline{\mathrm{W}}_{21}$ of $\mathrm{K}_{0}$ has less effect on power stiffness. Even if $\Omega$ increases, the coefficient $\dot{\theta}^{2}, \ddot{\theta}$ in front of $\overline{\mathrm{W}}_{22}, \overline{\mathrm{W}}_{21}$ increases, and its effect on dynamic stiffness is still small, and the "softening" effect of deformation coupling is not obvious.

2. When the radius of the center is $R=1 \mathrm{~m}$, the coupling of velocity and angular velocity increases the added stiffness by the formula (8), (12), (14). When the flexible beam has no initial deformation, the initial conditions is $\mathrm{q}_{f}=0, \dot{\mathrm{q}}_{f}=0$. Given different stable speeds $\Omega=2 \mathrm{rad} / \mathrm{s}, \Omega=4 \mathrm{rad} / \mathrm{s}$, by zeroorder approximate model, one-order coupling model and the precise coupling model point deformation displacement is respectively obtained in this paper.

3. When the radius of the center is $R=2 \mathrm{~m}$, the coupling of velocity and angular velocity increases the added stiffness by the formula (8), (12), (14). When the flexible beam has no initial deformation, the initial conditions is $\mathrm{q}_{f}=0, \dot{\mathrm{q}}_{f}=0$. Given different stable speeds $\Omega=2 \mathrm{rad} / \mathrm{s}$, $\Omega=4 \mathrm{rad} / \mathrm{s}$, by using zero-order approximate model, one-order coupling model and the precise coupling model point deformation displacement is respectively obtained in this paper.

4. When the end of the flexible beam has initial deformation, such as satellite antenna by solar wind, space temperature change, assume that $R=1 \mathrm{~m}$, in the $\mathrm{e}_{2}^{1}$ direction deformation displacement of $V=0.1 \mathrm{~m}, 0.2 \mathrm{~m}$, then the displacement $\mathrm{q}_{f 0}$ of the nodes is first evaluated by using ANSYS software. Given the different steady speed $\Omega=2 \mathrm{rad} / \mathrm{s}, \Omega=4 \mathrm{rad} / \mathrm{s}$, the deformation displacement of the end points is calculated by using one-order coupling model and the precise coupling model in this paper.

Frequency analysis of the above model is carried out. Some of the literature is based on the dynamic equation of the zero-order approximate model, and the influence of the gyro $G$ is not considered when calculating the natural frequency of the rotary beam. The coupling of deformation was considered by applying one-order coupling model, in addition this paper accurate model in generalized stiffness matrix $K$, generalized force matrix $Q$, has contained the coupling term of the basis points angular velocity and speed. These factors have a certain effect on the natural frequency. According to the literature [4], we set $\mathrm{q}_{f 0}$ to the static deformation relative to the body frame $\mathrm{e}^{1}$ under generalized force $\mathbf{Q}, \mathbf{q}_{f 1}$ is the displacement of the micro amplitude of the relative equilibrium position.

$$
\begin{aligned}
& \boldsymbol{q}_{f}=\boldsymbol{q}_{f 0}+\boldsymbol{q}_{f 1} \\
& \boldsymbol{K} \boldsymbol{q}_{0}=\boldsymbol{Q}
\end{aligned}
$$

The vibrational equation of the beam is obtained.

$$
\boldsymbol{M} \ddot{\boldsymbol{q}}_{f 1}+\boldsymbol{G} \dot{\boldsymbol{q}}_{f 1}+\boldsymbol{K} \boldsymbol{q}_{f 1}=\boldsymbol{O}
$$

In the state space $\boldsymbol{Y}=\left[\begin{array}{ll}\dot{\boldsymbol{q}}_{f 1} & \boldsymbol{q}_{f 1}\end{array}\right]^{T}$, the above equation is rewritten.

$$
\boldsymbol{M}_{z} \dot{\boldsymbol{Y}}+\boldsymbol{K}_{z} \boldsymbol{Y}=\mathbf{0}
$$




$$
\boldsymbol{M}_{z}=\left[\begin{array}{cc}
\boldsymbol{M}_{q} & \boldsymbol{0} \\
\boldsymbol{0} & \boldsymbol{I}
\end{array}\right], \boldsymbol{K}_{z}=\left[\begin{array}{cc}
\boldsymbol{G}_{q} & \boldsymbol{K}_{q} \\
-\boldsymbol{I} & \boldsymbol{0}
\end{array}\right]
$$

Let $\mathrm{Y}=\overline{\mathrm{Y}} e^{p t}$, the eigenvalue equation for these problems is

$$
\left(p \boldsymbol{M}_{z}+\boldsymbol{K}_{z}\right) \overline{\boldsymbol{Y}}=\mathbf{0}
$$

\section{Analysis}

When $R=0 \mathrm{~m}, \mathrm{~K}_{1}$ are the same by using three models, but considering the intercoupling of vertical and horizontal deformation, as well as the distortion mode of corresponding points in the middle line is replaced by any deformation pattern In the horizontal, $\mathrm{K}_{0}$ is different. With the increase of steady speed, the accurate model of each order natural frequency is a slightly increased coupling model. The coupling effect makes the dynamic stiffness increase, and two models of each order natural frequency are increased with the increase of rotational speed increases. All this reflects dynamic stiffening.

When $R \neq 0$, Because of the coupling of the velocity and the angular velocity of base point, the $\mathrm{K}_{1}$ of the precise model of this paper increases relative to one-order coupling model. Due to the increase of the additional stiffness term, under the different rotating speed and radius of centrosome, endpoint deformation displacement of the precise model is reduced relative to one-order coupling model. Increased with the increase of centrosome radius or steady speed, when the movement is stable, the deformation amplitude of the precise model slightly larger than one-order coupling model, and gradually tend to be one-order coupling model. As the coupling of speed and angular velocity increases, it is less than one-order coupling model; the frequency of the precise model is slightly more than one-order coupling model. With the increase of steady speed, the inherent frequency difference between the two models increases gradually. In this paper, the precise model of various frequencies are higher than that of one-order coupling model. The coupling effect of velocity and angular velocity of base point also make the beam tends be "rigid". Its role cannot be ignored in basis points movement at high speeds.

No additional stiffness $\mathrm{K}_{1}$ is considered in zero-order approximate model, so Whether $\mathrm{R}$ is zero, whether or not it has an initial deformation, when calculating the endpoint deformation, the calculation results are divergent when the steady speed increase; The frequency of its various frequencies decreases with the increase of the speed, and the base frequency is unsolved after the steady turn of the revolution, and does not reflect dynamic stiffening; In the case of zero-order approximate model, the gyro $G$ makes the natural frequency slightly lower. The frequencies of zeroorder approximate model are lower than one-order coupling model and the present exact model.

With the increase of the radius of the central body and the increase of rotational speed, the frequency difference of the two models also increases correspondingly; When $R=0 \mathrm{~m}$, the speed and acceleration of the base point are zero, no point velocity and angular velocity coupling, and only additional deformation coupling term, frequency difference of the two models is very small. The addition of $\mathrm{R}$ or $\Omega$ increases the Stiffness term of the precision model, which makes the frequency of the precise model higher than one-order coupling model.

From the above analysis shows, for a planar flexible beam, consider fully the geometric nonlinear deformation, deformation coupling model to increase did not produce significant softening effect, on the contrary make beam slightly "rigid". At the same time by using the precision model, the new coupling term of velocity and angular velocity of the base point will influence the simulation when the speed of motion is larger.

\section{Conclusion}

This paper studies the dynamic properties of the plane flexible beam under the non-inertial coordinate system known to the law of motion. The research results show that for the planar flexible beam, consider fully the geometric nonlinear deformation, deformation coupling model to increase did not produce significant softening effect, on the contrary make beam slightly "rigid". At the same 
time, under the accurate model, the basis of new velocity and angular velocity of the coupling term, under the condition of velocity is larger for the calculation of model simulation.

In this paper, the plane flexible beam under the non-inertial coordinate system is studied, and the influence of the deformation coupling term and the coupling effect of velocity and angular velocity of base point on different structures are discussed. For planar flexible beam structure, the deformation deformation coupling term and the coupling effect of velocity and angular velocity of base point have the effect of "rigidification" on the beam, but the simple deformation coupling is small.

For flexible beam with initial deformation, whether using zero-order approximate model, oneorder coupling model and the present exact model, the deformation and movement are larger volatility. Even if the rigid motion is constant, the movement of deformation still fluctuates greatly. This indicates that the structure should avoid initial deformation or some control strategy should be adopted.

\section{References}

[1]. He Xingsuo, Deng Fengyan, Wu Genyong. Dynamic modeling of a flexible beam with large overall motion and nonlinear deformation using the finite element method. Acta Physica Sinica, Vol. 59 (2010) No. 1, p. 25-29.

[2]. Deng Fengyan, He Xingsuo, Li Liang, et al. Dynamics modeling for a rigid-flexible coupling system with nonlinear deformation field. Multibody System Dynamics. Vol. 18 (2007) No. 4, p. 559-578.

[3]. He Xingsuo, Deng Fengyan, Wang Rui. Exact dynamic modeling of a spatial flexible beam with large overall motion and nonlinear deformation. Acta Physica Sinica. Vol. 59 (2010) No. 3, p. 1428-1436.

[4]. He Xingsuo, Li Xuehua, Deng Fengyan. Analysis and imitation of dynamic properties for rigidflexible coupling systems of a planar flexible beam. Acta Physica Sinica. Vol. 60 (2011) No. 2: 024502.

[5]. Yang Hui: Study on dynamic modeling theory and experiments for rigid-flexible coupling systems (Doctor degree, Shanghai Jiao Tong University, China, 2002). p. 45-76.

[6]. P. SHI, J. McPHEE, G.R. HEPPLER. A Deformation Field for Euler-Bernoulli Beams with Applications to Flexible Multibody Dynamics. Multibody System Dynamics. Vol. 5 (2001) No. 1, p. 79-104. 